Swarthmore College

Works

$1-1-2016$

\title{
Efficient Image Processing Via Compressive Sensing Of Integrate- And-Fire Neuronal Network Dynamics
}

\author{
Victor J. Barranca \\ Swarthmore College, vbarran1@swarthmore.edu \\ G. Kovačič \\ D. Zhou \\ D. Cai
}

Follow this and additional works at: https://works.swarthmore.edu/fac-math-stat

Part of the Mathematics Commons

Let us know how access to these works benefits you

\section{Recommended Citation}

Victor J. Barranca, G. Kovačič, D. Zhou, and D. Cai. (2016). "Efficient Image Processing Via Compressive Sensing Of Integrate-And-Fire Neuronal Network Dynamics". Neurocomputing. Volume 171, 1313-1322. DOI: 10.1016/j.neucom.2015.07.067

https://works.swarthmore.edu/fac-math-stat/164

This work is brought to you for free by Swarthmore College Libraries' Works. It has been accepted for inclusion in Mathematics \& Statistics Faculty Works by an authorized administrator of Works. For more information, please contactmyworks@swarthmore.edu. 


\title{
Efficient Image Processing Via Compressive Sensing of Integrate-And-Fire Neuronal Network Dynamics
}

\author{
Victor J. Barranca ${ }^{\mathrm{a}, \mathrm{b}, \mathrm{c}}$, Gregor Kovačičd ${ }^{\mathrm{d}}$, Douglas Zhou ${ }^{\mathrm{e}, * *}$, David Cai ${ }^{\mathrm{a}, \mathrm{b}, \mathrm{e}, *}$ \\ ${ }^{a}$ Courant Institute of Mathematical Sciences 8 Center for Neural Science, New York \\ University, New York, NY 10012, USA \\ ${ }^{b}$ NYUAD Institute, New York University Abu Dhabi, PO Box 129188, Abu Dhabi, UAE \\ ${ }^{c}$ Department of Mathematics and Statistics, Swarthmore College, 500 College Avenue, \\ Swarthmore, PA 19081, USA \\ ${ }^{d}$ Mathematical Sciences Department, Rensselaer Polytechnic Institute, 110 8th Street, \\ Troy, NY 12180, USA \\ ${ }^{e}$ Department of Mathematics, MOE-LSC, and Institute of Natural Sciences, Shanghai \\ Jiao Tong University, Shanghai 200240, China
}

\begin{abstract}
Integrate-and-fire (I\&F) neuronal networks are ubiquitous in diverse image processing applications, including image segmentation and visual perception. While conventional I\&F network image processing requires the number of nodes composing the network to be equal to the number of image pixels driving the network, we determine whether I\&F dynamics can accurately transmit image information when there are significantly fewer nodes than network input-signal components. Although compressive sensing (CS) theory facilitates the recovery of images using very few samples through linear signal processing, it does not address whether similar signal recovery techniques facilitate reconstructions through measurement of the nonlinear dynamics of an I\&F network. In this paper, we present a new framework for recovering sparse inputs of nonlinear neuronal networks via compressive sensing. By recovering both one-dimensional inputs and two-dimensional images, resembling natural stimuli, we demonstrate that input information can be well-preserved through nonlinear I\&F network dynamics even when the number of network-output measurements is significantly smaller than the number of input-signal components. This work suggests an important exten-
\end{abstract}

${ }^{*}$ cai@cims.nyu.edu

${ }^{* *} z d z @ s j t u . e d u . c n$ 
sion of CS theory potentially useful in improving the processing of medical or natural images through I\&F network dynamics and understanding the transmission of stimulus information across the visual system.

Keywords:

Compressive sensing; Neuronal networks; Signal processing; Nonlinear Dynamics

\section{Introduction}

The recent emergence of compressive sensing (CS) has in many ways transformed the field of signal processing, giving rise to a multitude of theoretical extensions and practical applications [1-6]. Especially ubiquitous in improving the accuracy of detailed signal reconstructions and formulating effective sampling schemes, applications of CS have emerged in diverse disciplines, including physics, biology, and image science [7-12]. According to CS theory, signals that have few dominant components in an appropriate domain can be reconstructed from a relatively small number of non-uniformly-spaced samples, yielding a sampling rate significantly lower than suggested by conventional signal processing theory [3]. The current theory of CS has mainly provided a framework for linear signal processing.

In this work, we discuss a new direction for compressive sensing that generalizes its application to signal processing in nonlinear integrate-and-fire (I\&F) neuronal network dynamics [13-16]. Integrate-and-fire models have proven useful in studying a diverse array of natural phenomena, including earthquake activity [17], insect communication [18, 19], and neuronal dynamics [20-22]. With regard to image processing in particular, I\&F networks are applicable to a variety of image segmentation problems, involving the grouping of image pixels by common features, such as gray-scale, color, or texture [23-25]. Similarly, I\&F networks have also been applied in edge detection, feature recognition, and image denoising [26-32]. A common characteristic of these I\&F network applications in image processing is that the same number of nodes compose the network as pixels in the input image, such that each node in the network is driven by a unique pixel.

Since images, such as visual stimuli and medical scans, are typically sparse in some domain [33], is it possible for CS theory to facilitate more efficient image processing through I\&F network dynamics by utilizing fewer nodes than pixels? To answer this question, we determine whether input signal informa- 
tion is well-preserved through the activity of a pulse-coupled I\&F neuronal network with significantly more input components than nodes. While CS theory often makes it possible to recover the input into a static linear system with only few output measurements, the problem of recovering the same input signal in nonlinear dynamical systems, such as neuronal networks, is more complicated. We resolve this difficulty by deriving a linear input-output relationship embedded in I\&F network dynamics, which is applicable to CS analysis. First, we demonstrate that we can use probabilistic arguments to derive an approximate nonlinear input-output mapping for such a network. Then, we derive a linearization for this nonlinear mapping, and illustrate the robustness of this approximation. Using this linearization and the set of output measurements, we establish a methodology for reconstructing network inputs via CS. A schematic model of this recovery process is given in Fig. 1.

Considering both one-dimensional and two-dimensional inputs of various sizes, analogous to natural stimuli, we obtain accurate and recognizable reconstructions using our novel recovery process. Our work establishes that I\&F neuronal dynamics can indeed successfully encode input images even for networks in which the number of nodes is an order of magnitude smaller than the number of input components. Comparing signal reconstructions using network dynamics and static CS with the same number of random measurements as nodes in the corresponding I\&F network, we observe a similar level of accuracy and conclude that signal information is comparably wellpreserved through network dynamics, giving insight into the conservation of stimulus information across sensory networks.

The organization of the paper is as follows. In Section 2.1, we briefly introduce and summarize the basic aspects of compressive sensing theory. In Section 2.2, we describe the I\&F network model that we will subsequently consider and then derive in Section 2.3 the network mapping of system input to output. Next, in Section 2.4, we derive the linearization to which we then apply CS theory and describe the CS algorithm we will use to reconstruct the system input from a sequence of network output measurements. We reconstruct several input signals in Section 3 and compare these dynamicalsystem input reconstructions to corresponding static signal reconstructions using the same number of measurements as network nodes. Finally, in Section 4 , we discuss the ramifications of this work and possible directions for future research. 


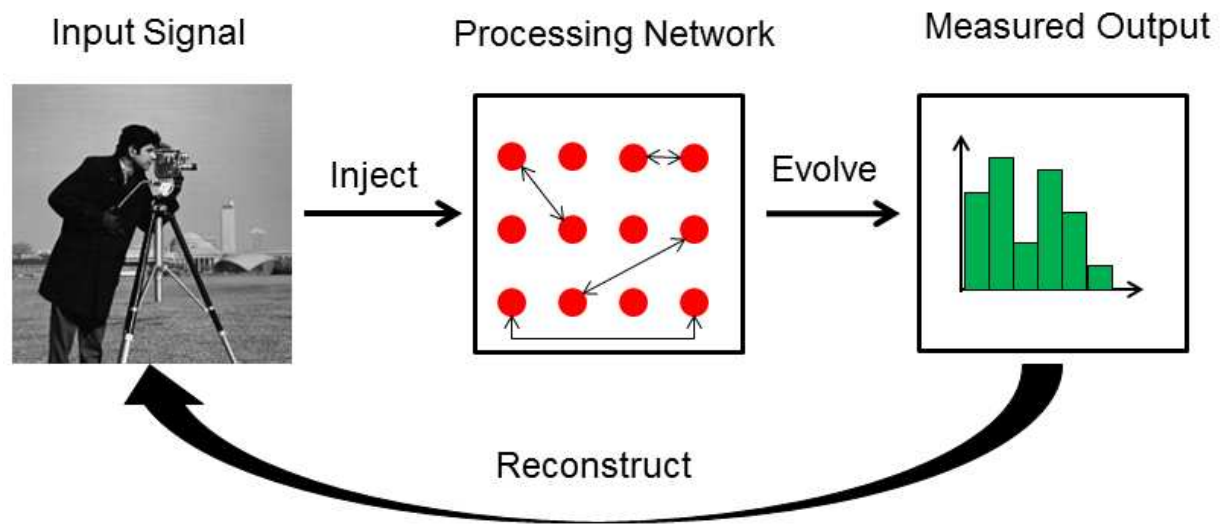

Figure 1: Schematic model of recovery process. A relatively large $n$-component signal is injected into a dynamical network for which we measure the response of $m$ nodes, where $m \ll n$. Based on these measurements, we reconstruct the original input signal using compressive sensing signal recovery based on a linear map intrinsic to the network dynamics.

\section{Methods}

\subsection{Compressive Sensing}

The Shannon-Nyquist theorem [34], underlying the sampling and compression of most current audio and visual signals, asserts that the sampling rate of a given signal depends on its bandwidth, $W$. According to the theorem, signals must be sampled at a rate of at least $2 W$ to guarantee successful reconstruction. In contrast, compressive sensing provides a simple alternative means for both efficiently sampling a broad class of signals and later reconstructing them with a high degree of accuracy. Using only the number of non-zero frequency components instead of the full range of frequencies to determine minimal sampling rates, compressive sensing demonstrates that it is possible to reconstruct signals with far fewer measurements than previously believed [1-3].

One of the main observations of CS theory is that since most signal components within a sufficiently large bandwidth frequently vanish in an appropriate domain, the sampling rate predicted by the Shannon-Nyquist theorem is typically too large. Such signals are considered sparse, since few of their components are non-zero. A signal with $n$ components is defined as $k$-sparse, with $k \ll n$, when at least one of its transforms into an appropriate space 
has at most $k$ components whose magnitude exceeds a small threshold $[1,3]$. For example, many commonly used signals, including both sound waves and images, are sparse in several domains [33]. For these signals, coordinate transformations, such as the Fourier, discrete cosine, and wavelet transforms, all typically yield sparse signal representations [35-37]. If an $n$-vector signal is $k$-sparse, then it is reasonable to expect that, since all data is contained in $k$ components, then the number of measurements necessary to determine the signal should be determined by $k$. Using this reasoning, optimally reconstructing a large signal from relatively few samples amounts to choosing the reconstruction with the most zero entries since such a signal is considered most compressible [2]. Remarkably, as long as the signal is sparse in at least one domain, the CS theory is applicable.

This signal recovery problem can be cast into the form of an underdetermined linear system. Suppose one seeks to recover an $n$-component signal, $x$, using only $m$ measurements, where $m \ll n$. In this case, one samples $x$ with an $m \times n$ measurement matrix, $R$, yielding $m$-component measured signal, $b$. In the case that $x$ is sparse, this problem is identical to solving the linear system

$$
R x=b .
$$

If $m<n$, there exists an infinity of $x$ 's one can choose from in selecting a viable reconstruction. In selecting the solution that best approximates the true sampled signal while using a minimal number of samples, a naive approach would be to choose the sparsest $x$. This is equivalent to minimizing the $\ell_{0}$ norm of $x$, which gives the number of non-zero entries of the signal. However, solving such an optimization problem for a large linear system is typically NP-hard and in most cases too computationally expensive. Assuming that $x$ is sufficiently sparse, compressive sensing theory demonstrates that minimizing $|x|_{\ell_{1}}=\sum_{i=1}^{n}\left|x_{i}\right|$ yields a reconstruction equivalent to finding the sparsest $x$ for a broad class of measurement matrices $[2,38]$. This specific $\ell_{1}$ minimization problem is identical to the widely studied linear programming problem

$$
\begin{gathered}
\text { minimize } y_{1}+\cdots+y_{n} \\
\text { given }-y_{i} \leq x_{i} \leq y_{i}, \quad i=1, \ldots, n,
\end{gathered}
$$


under constraint (1). Problems of this type can be efficiently solved through a variety of algorithms, including least angle regression (LARS), least absolute shrinkage and selection operator (LASSO), Polytope Faces Pursuit, and homotopy methods [39, 40].

It is important to remark that if the measured signal is not sparse in the sampled domain, but is instead sparse under a transform, $T$, then a similar linear system

$$
\phi \hat{x}=b,
$$

where $\phi=R T^{-1}$ and $\hat{x}=T x$ should instead be considered. By solving (3) for $\hat{x}$, the solution in the non-sparse domain, $x=T^{-1} \hat{x}$, can be computed subsequently.

With this framework, to complete the signal reconstruction procedure using CS, we need only select how best to sample the signal. Compressive sensing theory demonstrates that measurement matrices, $R$, which demonstrate mutual incoherence and the restricted isometry property (RIP) yield successful reconstructions with near certainty [2, 6, 38]. Intuitively, such matrices have uncorrelated columns and approximately preserve the size of sparse signals. More specifically, coherence gives a measure of correlation between entries of $R$ and is defined as $M(R)=\max \left\{\left|R_{i}^{T} R_{j}\right|: i \neq j\right\}$, such that $R=\left[R_{1} \ldots R_{n}\right]$ with $\left\|R_{i}\right\|_{2}=1$, for all $i=1 \ldots n$. Matrix $R$ is considered mutually incoherent if $M(R)$ is sufficiently small. In Ref. [41], for example, it is shown that if $|x|_{\ell_{0}}<(\sqrt{2}-0.5) / M(R)$ for a sparse $x$ that solves $(1)$, then the $\ell_{0}$ and $\ell_{1}$ norms are equivalent. Similarly, $R$ satisfies the RIP property if $\left(1-\delta_{k}\right)\|x\|_{2}^{2} \leq\|R x\|_{2}^{2} \leq\left(1+\delta_{k}\right)\|x\|_{2}^{2}$ for all $k$-sparse $x$ and some $\delta_{k} \in(0,1)$.

A large class of matrices exhibits these properties, most notably several types of random matrices with independent identically distributed elements. For example, matrices with either independent identically distributed Gaussian entries with mean 0 and variance $1 / n$, or Bernoulli distributed entries of value $\pm 1 / \sqrt{n}$ have both been shown to yield successful reconstructions with high probability $[1,2]$. In practice, experimentally verifying the success of a CS reconstruction is often the simplest approach to ascertaining the validity of a given measurement matrix.

\subsection{Network Model}

Using a pulse-coupled I\&F network model and the theory of CS, we present a new framework through which network dynamics can be used to 
recover network inputs with significantly more components than nodes composing the network. We note that our methodology is quite general and may also be applicable to other classes of networks, which we will discuss further in Section 2.3. The network model we consider is composed of two layers, an input layer and a processing layer. For ease of discussion, we describe this network in the terminology of a neuronal network, in which each node is considered to be a neuron. In the framework of neuroscience, this model roughly represents sensory receptors (input layer) and processing neurons (output processing layer), respectively. We model the network input layer by a vector with components determined by the input characteristics, corresponding to a sequence of post-synaptic currents in the setting of a neuronal network. Each input current is transmitted downstream to a number of processing neurons, whose voltage (output) dynamics are described by a current-based, integrate-and-fire (I\&F) model [13-15, 20, 21, 42].

According to our network model, the membrane-potential dynamics of the $i^{\text {th }}$ processing neuron is governed by the differential equation

$$
\tau \frac{d v_{i}}{d t}=-\left(v_{i}-V_{R}\right)+f \sum_{j=1}^{n} B_{i j} p_{j}+\frac{S}{N_{A}} \sum_{\substack{k=1 \\ k \neq i}}^{m} A_{i k} \sum_{l} \delta\left(t-\tau_{k l}\right),
$$

evolving from the reset potential $V_{R}$ until it reaches the threshold potential $V_{T}$. At the $l^{\text {th }}$ time this occurs, $\tau_{i l}$, we say that this neuron has fired (or spiked), reset $v_{i}$ to $V_{R}$, and inject the currents $\left(S / N_{A}\right) \delta\left(t-\tau_{i l}\right)$ into all the other processing neurons post-connected to (neighboring) it, with $\delta(\cdot)$ being the Dirac delta function. The $j^{\text {th }}$ neuron is considered to be post-connected to the $i^{\text {th }}$ neuron if $A_{j i} \neq 0$. Moreover, $\tau$ is the membrane-potential time-scale, $n$ and $m$ are the numbers of the input and processing neurons respectively, $p=\left(p_{1}, \ldots, p_{n}\right)$ are the signal strengths transmitted by the input neurons, $B=\left(B_{i j}\right)$ and $A=\left(A_{i j}\right)$ are connection matrices between the input and processing neurons and between processing neuron pairs respectively, $f$ and $S$ are the respective overall strengths of those connections, and $N_{A}$ is the number of connections between pairs of processing neurons.

The architecture of our network model can be described with a graphtheoretic structure. Similar network models involving sets of time-evolving agents have been used in a number of applications [13, 43-53]. In describing network connectivity, we say that the $j^{\text {th }}$ neuron, or node, is post-connected to (neighboring) the $i^{\text {th }}$ neuron if a directed edge connects the $i^{\text {th }}$ neuron to the $j^{\text {th }}$ neuron and therefore $A_{j i} \neq 0$. When the $i^{\text {th }}$ neuron fires, the $j^{\text {th }}$ 
neuron will thus receive a voltage jump of size $A_{j i} S / N_{A}$.

Motivated by the random sampling strategy typical in CS theory, we assume that every processing neuron samples the input signal randomly, and also that processing neurons are connected to each other randomly. Therefore, we take the elements of the connectivity matrices $A$ and $B$ to be Bernoulli-distributed with respective non-zero values 1 and $1 / N_{B}$, where $N_{B}$ is the number of connections between input and processing neurons found in $B$. Specifically, in our model $A_{i j}=1$ with probability 0.05 and $B_{i j}=1 / N_{B}$ with probability 0.001 , reflecting the commonly observed sparse connectivity in local cortical circuits and sensory systems [54-56]. From an engineering standpoint, this assumption may instead reflect the use of only a small amount of data per signal recovery. Nevertheless, we remark that a broad class of matrices with independent identically distributed elements may also be appropriate for alternative applications. Likewise, in formulating a more general or different network model, the processing neurons need not even be connected to one another, and may rather respond to the input signal independently. To examine whether efficient CS reconstructions are still feasible utilizing nonlinear network dynamics, we choose the numbers $n$ of the input components and $m$ of the output neurons to be such that $n \gg m$. Using this network framework, we demonstrate how the measured output of only the $m$ nodes can be used to recover an $n$-component input. In the following analysis, we first consider the network input, $p$, to be a one-dimensional wave and later consider a two-dimensional grey-scale image.

We simulate this model using an event-driven algorithm in which we analytically solve for neuronal voltages and spike times, choosing the dimensionless potential values $V_{R}=0$ and $V_{T}=1, n=10^{4}, m=10^{3}, S=1$, and $f=1$ for numerical simulations unless stated otherwise $[57,58]$. Consistent with realistic neuronal simulations, we choose time-scale $\tau=20 \mathrm{~ms}$ [59-61], and a total simulation time of $t_{f}=200 \mathrm{~ms}$, which is of the same order as human reaction-time for visual stimuli $[62,63]$.

\subsection{Coarse-Graining}

To determine whether input signals are well transmitted and preserved through network dynamics, we perform a coarse-graining procedure using probabilistic arguments to obtain a mapping between network input and output. Typically the underlying assumption of coarse-graining is that one can approximate network dynamics by forming groups of nodes with statistically averaged input responses in each group [64, 65]. Here, we do not 
make this assumption, but instead use similar ideas to derive an analytical expression for the firing rates of individual processing neurons described by an $m$-vector, $\mu$.

In carrying out our coarse-graining, we consider a statistical ensemble of nearly identical networks of processing neurons, differing only in initial voltages, $v_{j}(t=0)$, and consequently input currents, $I_{j}$, for $j=1, \ldots, m$. For each realization of the network in the ensemble, the $j^{\text {th }}$ neuron is injected with a new independent spike train of pulses transmitted by pre-connected neurons in addition to an input current, $f \sum_{j=1}^{n} B_{i j} p_{j}$, for $i=1, \ldots, m$. Since these spike trains correspond to firing events received within the network of processing neurons, the input rate, $r_{j}$, is equal to the rate at which the $j^{\text {th }}$ neuron in the network receives spikes from neurons pre-connected to it. Moreover, for each realization of the network in the ensemble, the connectivity matrices $A$ and $B$ are initially generated with each containing independent identically distributed Bernoulli entries and remain the same throughout each realization.

We further assume that the network undergoes a large number of firing events, of which each only evokes a small voltage jump to post-connected neurons. In other words, we make the assumption of high spike frequency and small spike magnitude, $\mu_{j} \rightarrow \infty$ and $S / N_{A} \rightarrow 0$, respectively. Under this assumption, the total network input to each processing neuron, say the $i^{\text {th }}$,

$$
\frac{S}{N_{A}} \sum_{\substack{k=1 \\ k \neq i}}^{m} A_{i k} \sum_{l} \delta\left(t-\tau_{k l}\right),
$$

becomes approximately a Poisson spike train [66]. As a consequence of this assumption, we find that, statistically, the effect of the network drive becomes the same as that of an approximately mean drive from pre-connected neurons,

$$
\frac{S}{N_{A}} \sum_{\substack{k=1 \\ k \neq i}}^{m} A_{i k} \mu_{k}
$$

Replacing the last term on the right hand side of (4) by (5), we obtain the membrane-potential solution 


$$
\begin{aligned}
v_{i}(t)= & v_{i}\left(t_{0}\right) e^{-\left(t-t_{0}\right) / \tau}+\left(1-e^{-\left(t-t_{0}\right) / \tau}\right) \\
& \times\left(V_{R}+f \sum_{j=1}^{n} B_{i j} p_{j}+\frac{S}{N_{A}} \sum_{\substack{k=1 \\
k \neq i}}^{m} A_{i k} \mu_{k}\right) .
\end{aligned}
$$

Letting $v\left(t_{0}\right)=V_{R}$ and $v\left(t_{0}+1 / \mu_{i}\right)=V_{T}$, where $\mu_{i}$ is the firing rate of the $i^{\text {th }}$ neuron, and dividing by the factor $1-e^{-1 / \tau \mu_{i}}$, we obtain the mapping

$$
\sum_{j=1}^{n} f B_{i j} p_{j}=\frac{V_{T}-V_{R}}{1-e^{-1 / \tau \mu_{i}}}-\frac{S}{N_{A}} \sum_{\substack{k=1 \\ k \neq i}}^{m} A_{i k} \mu_{k},
$$

which holds for each individual processing neuron in the network. It is important to remark that for other network models, similar arguments may also apply, assuming it is possible to quantify the time-averaged output of each node and also derive an approximation of the mean interactions between nodes. For more general models with additional output variables or more complex nonlinear node interactions, the coarse-graining methodology presented in Refs. [64, 65] may also be applicable in obtaining a network input-output mapping.

\subsection{Network Input-Output Relationship}

While (7) does indeed give us an input-output relationship that we can use to reconstruct the input signal using output firing rates of the processing neurons, it is nonlinear with respect to $\mu$ and may also be nonlinear with respect to $p$ for more general network models. In our particular case, once $\mu$ is computed from simulation of I\&F network dynamics, the right-hand side of (7) is completely determined, giving an underdetermined linear system in network input, $p$. We remark that while linearization is not necessary for this particular I\&F network model since (7) is linear with respect to $p$, for more general and complex models, one may have to perform linearization in order to apply CS theory. Therefore, we also consider the linearization of (7) for the problem of input image recovery.

To successfully encode the input signals transmitted by the input neurons, we assume the primary drive for the processing neurons in the network is the large external input, $B p$. In this case, the network will be in a high-firingrate dynamical regime. Therefore, we perform Taylor expansion with respect 
to the small parameter $1 /\left(\mu_{j}\right)$ and up to the leading order $O\left(1 /\left(\mu_{j}\right)^{2}\right)$, we obtain

$$
f \sum_{j=1}^{n} B_{i j} p_{j}=\tau \mu_{j}\left(V_{T}-V_{R}\right)+\frac{\left(V_{T}-V_{R}\right)}{2}-\frac{S}{N_{A}} \sum_{\substack{k=1 \\ k \neq i}}^{m} A_{i k} \mu_{k} .
$$

With respect to the entire network of processing neurons, rewriting (8), we obtain the $m$-dimensional linear system approximation for all firing rates over the entire processing neuron network

$$
f B p=\left(\tau \mu+\frac{L}{2}\right)\left(V_{T}-V_{R}\right)-\frac{S}{N_{A}} A \mu,
$$

where $L$ denotes an $m$-vector of ones.

While the linearization given by (9) is only valid if the network input is sufficiently large, using our explicit solution for external drive strength in (7), we can easily verify the regime of validity for this linearization. In Fig. 2 (a), we plot the network gain curves, which give the network-averaged firing rate as a function of the input signal strength. We compare the computed firing rates using direct full-model simulation, given by (4), to the theoretically computed firing rates predicted with both the nonlinear map given by (7) and the linearization in (9). For sufficiently large input-signal strengths, we observe close agreement between the network-averaged firing rates computed using the simulated full-model and both the non-linear and linear inputoutput mappings, yielding a large dynamical regime in which the theoretical approximations are valid.

\subsection{Firing Rate Recovery}

While our ultimate goal is to use the linear mapping in (9) to reconstruct the network input signal using the network output firing rates, it is important to note the simpler problem of computing network output as a function of the input signal can also be solved with this linearization. Given knowledge of the input signal, $p$, as well as the network architecture prescribed by connectivity matrices $A$ and $B$, this is a problem of solving the following linear system

$$
\left(\tau\left(V_{T}-V_{R}\right) I-\frac{S}{N_{A}} A\right) \mu=f B p-\frac{L\left(V_{T}-V_{R}\right)}{2}
$$

for the unknown $m$-vector $\mu$, where $I$ is the $m \times m$ identity matrix. Since the linear system is $m \times m$ in this case, a unique solution can be computed using 
standard numerical linear algebra solution techniques. We remark that since the entries of the matrix given by $S A /\left(N_{A} \tau\left(V_{T}-V_{R}\right)\right)$ are small relative to the order-unity diagonal entries of $I$, the problem will not be singular. Hence, in the regime of validity of our linearization, we expect our theoretical prediction of $\mu$ to closely match the results of numerical simulation of the network model (4). In Fig. 2 (b), we plot the individual processing neuron firing rates, $\mu_{j}$, measured from both numerical simulation and solution of (10). For comparison, we also include the firing rates obtained by solving nonlinear system (7) for $\mu$ using iteration with respect to fixed-points [67]. We observe that even on the fine scale of individual processing-neuron output, all three approaches yield nearly identical results for a large range of $f$.

\subsection{Compressive Sensing Network Input Signal Recovery}

With our linearization defined in (9), we now seek to recover an input signal, $p$, using only the output firing rates of the processing neurons measured through numerical simulation, $\mu$, and the network architecture, as encoded by the connectivity matrices $A$ and $B$. To apply compressive sensing in recovering a sparse representation of a two-dimensional $p$, we consider the vectorization of the two-dimensional discrete cosine transform of the input signal matrix, $\hat{p}=\left(\hat{p}_{1}, \ldots, \hat{p}_{n}\right)=(D \otimes D) p$, where $p$ is vectorized, $\otimes$ denotes the $n \times n$ Kronecker product

$$
D \otimes D=\left[\begin{array}{ccc}
D_{11} D & \cdots & D_{1 \sqrt{n}} D \\
\vdots & \ddots & \vdots \\
D_{\sqrt{n} 1} D & \cdots & D_{\sqrt{n} \sqrt{n}} D
\end{array}\right]
$$

$D$ is the $\sqrt{n} \times \sqrt{n}$, one-dimensional discrete cosine transform matrix with entries

$$
D_{i j}=\left(D^{-1}\right)_{i j}^{T}=\omega(i) \cos \left(\frac{(i-1)(2 j-1) \pi}{2 \sqrt{n}}\right),
$$

$\omega(1)=(1 / n)^{1 / 4}$, and $\omega(i \neq 1)=(4 / n)^{1 / 4}$. In solving the related problem of recovering $\hat{p}$, the linear model we consider is 
(a)

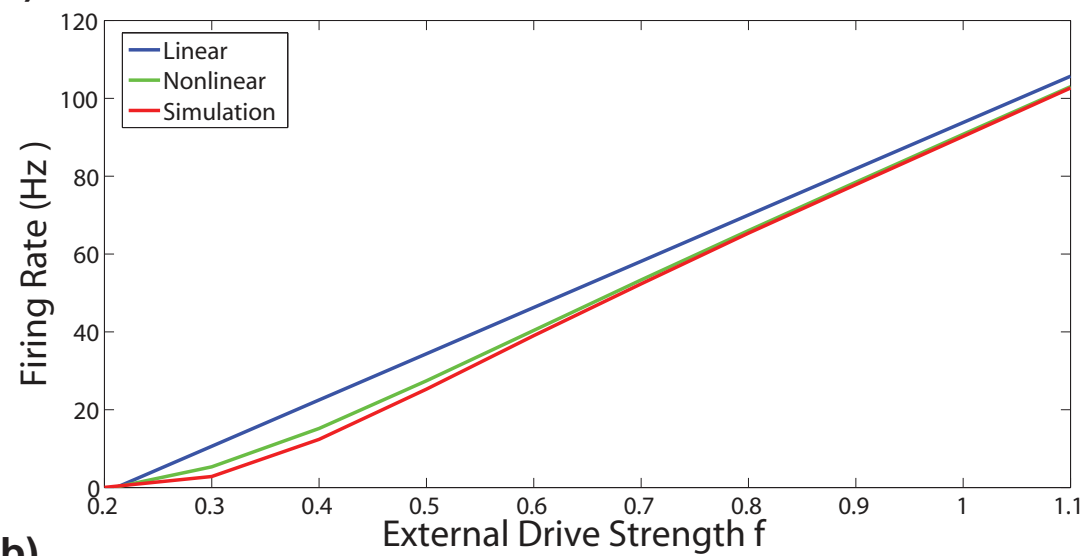

(b)

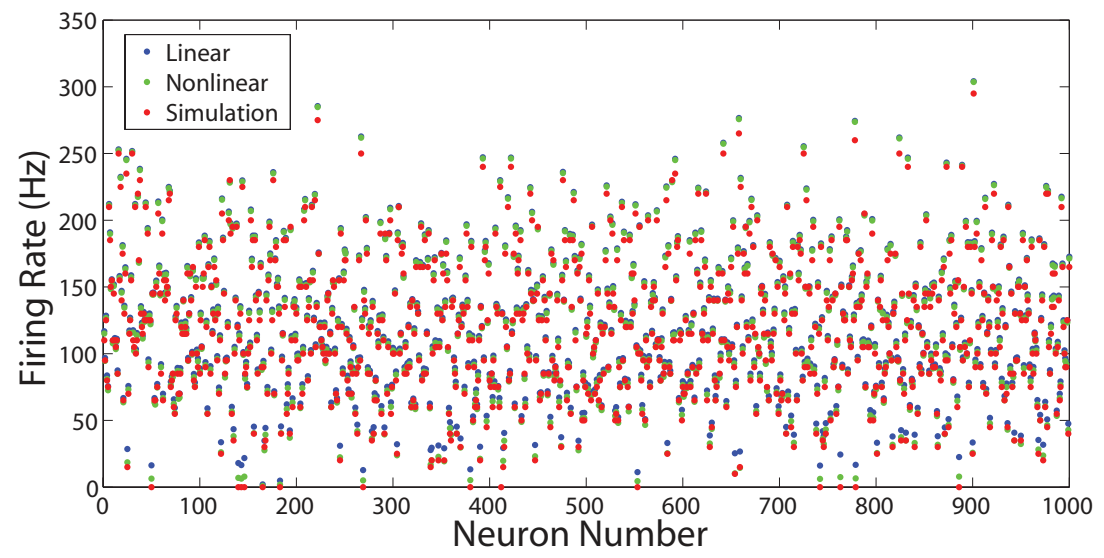

Figure 2: Closeness of the firing rates produced by the I\&F model and firing-rate mapping for each processing neuron in the network. The input signal used is the $100 \times 100$ pixel representation of the image presented in Fig. 5 (i). (a) Dependence of the networkaveraged firing rate on the input signal strength, $f$, computed using direct simulation of the I\&F model (4) (red), the nonlinear input signal approximation given by (7) (green), and the linearization in (9) (blue) (b) Comparison of individual processing neuron firing rates using the same color scheme as in (a). Comparing the network-averaged firing rates for each case when $f=1$, we observe that there is particularly close agreement between the firing rates of individual neurons, with many overlapping points.

$$
\begin{aligned}
& f \sum_{j=1}^{n} B_{i j}(D \otimes D)_{i j}^{-1} \hat{p}_{j}=\left(\tau \mu_{i}+\frac{1}{2}\right)\left(V_{T}-V_{R}\right) \\
&-\frac{S}{N_{A}} \sum_{\substack{k=1 \\
k \neq i}}^{m} A_{i k} \mu_{k} . \\
& 13
\end{aligned}
$$


Applying the CS theory discussed in Section 2.1, since the discrete cosine transform of the stimulus, $\hat{p}$, is sparse, recovering $\hat{p}$ is reduced to minimizing the sum $\sum_{j=1}^{n}\left|\hat{p}_{j}\right|$ under the constraint (11). Solving this minimization problem is equivalent to solving the $L_{1}$ optimization problem (2) under the constraint (11) where $x_{i}=\hat{p}_{i}$. We solve this optimization problem with a greedy algorithm known as the Orthogonal Matching Pursuit [39]. Once $\hat{p}$ is recovered, we finally invert the two-dimensional discrete cosine transform and the vectorization to obtain the input signal $p$. Note that once the input image recovery problem is posed in the form of (11), the optimization problem becomes solvable with the one-dimensional CS techniques discussed in Section 2.1. In the case of a one-dimensional input signal, we need only consider the one-dimensional discrete cosine transform of $p$ rather than the vectorized two-dimensional analogue, with the remaining details of the reconstruction unchanged. We summarize the input signal recovery process for two-dimensional input images processed through I\&F network dynamics in the following algorithm.

\section{Compressive Sensing Input Image Recovery Algorithm}

1. Simulate the model network according to (4) and record firing rates $\mu$ of processing neurons.

2. Solve the optimization problem (2) under the constraint (11) such that $x_{i}=\hat{p}_{i}$, where $\hat{p}_{i}$ is the two-dimensional discrete cosine transform of the input-image pixel matrix.

3. Compute the reconstructed input signal $p=(D \otimes D) \hat{p}$ from recovered sparse representation $\hat{p}$, and invert the vectorization of the recovered signal to obtain the image pixel matrix.

\section{Results}

\subsection{One-Dimensional Input Signal Recovery}

To demonstrate the robustness of this CS signal recovery procedure, we apply our methodology using both one-dimensional and two-dimensional input signals. In both cases we consider networks with a $10: 1$ ratio between input neurons and processing neurons, yielding a factor of 10 reduction in the number of output samples. Later, we will compare our results when the reduction factor is reduced and also when the input signal size is increased. We first consider a one-dimensional input signal of the form 
Table 1: One-Dimensional Input Signal Parameters

\begin{tabular}{|c||c|}
\hline Parameter & Value \\
\hline$a_{0}$ & 3000 \\
\hline$a_{1}$ & 600 \\
\hline$f_{1}$ & 0.5 \\
\hline$a_{2}$ & 600 \\
\hline$f_{2}$ & 2 \\
\hline$a_{3}$ & 600 \\
\hline$f_{3}$ & 4 \\
\hline$a_{4}$ & 0.06 \\
\hline$f_{4}$ & 20 \\
\hline
\end{tabular}

$$
p_{\text {cont }}(x)=a_{0}+\sum_{i} a_{i} \cos \left(f_{i} x\right)
$$

where $x$ is the spatial location of the input, determined by the index of a given input neuron, $a_{i}$ is the amplitude of constituent wave-form $i$, and $f_{i}$ is the corresponding frequency of the wave. The parameters of the signal are listed in Table 1, and chosen such that the signal contains several lowfrequency, moderate-amplitude terms and one noise-like, high-frequency, lowamplitude term. This composition is chosen such that the signal is sparse in the frequency domain, yet mimics a portion of the noise realistically present in many radio-wave or sound-wave signals.

To determine the above spatially oscillatory signal injected into any given processing neuron in the network, we discretize the input such that $p_{j}=$ $p_{\text {cont }}\left(\frac{j}{1000}\right)$. The input signal therefore consists of uniformly spaced samples of the original continuous signal on the $x$ interval $[0,10]$. Injecting this signal into our model network for $t=200 \mathrm{~ms}$, we plot in Fig. 3 the original input signal along with the corresponding network CS reconstruction using (9). We observe close agreement, with the various compressive sensing reconstructions clearly clustered around the injected signal. The reconstructions from the network output closely follow the shape of the input signal, with a relatively small scatter around the solution as a consequence of information that cannot be readily revealed through compressive sensing of network dynamics, which we investigate further in the following analysis.

Using the same sampling scheme as in the network-dynamics CS recovery via (9), we plot for comparison the static CS signal reconstruction and also 


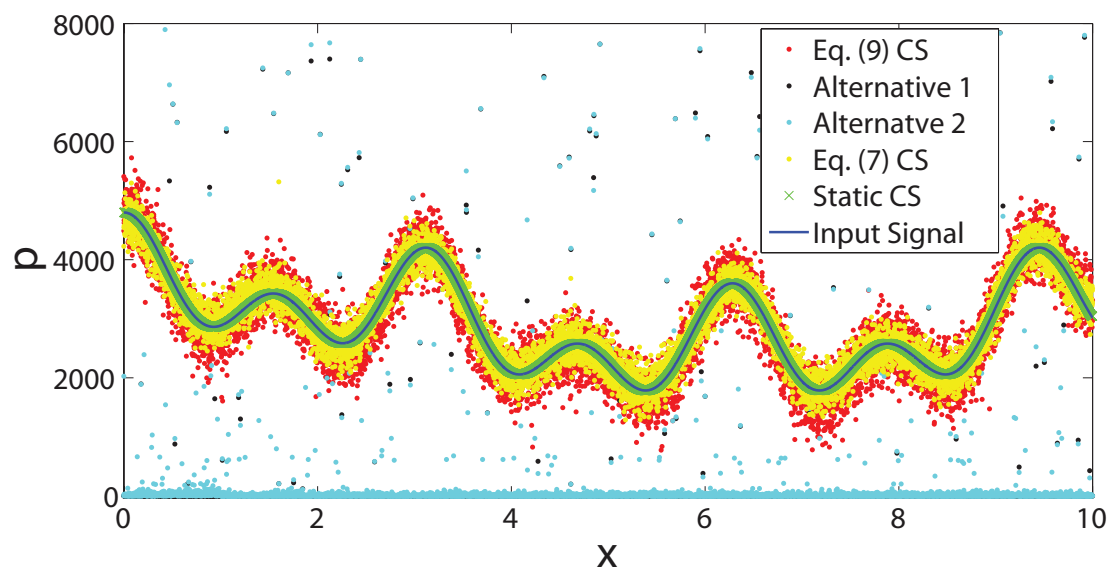

Figure 3: Reconstruction of one-dimensional input signal. Comparison of the original onedimensional input signal (blue), defined by (12), to several reconstructions: network CS reconstructions using (9) (red) and (7) (yellow), static CS reconstruction (green), and two alternative reconstructions, obtained by solving (9) directly using Gaussian elimination (black and cyan). The reconstruction errors, defined by (13), for the network CS reconstruction using Eqs. (9) and (7) are 0.1015 and 0.0671 respectively. The corresponding error for the static CS reconstruction is 0.0004. Each simulated network is composed of $n=10000$ input neurons and $m=1000$ processing neurons. We choose the connection probability of $A$ to be 0.05 and $B$ to be 0.001 .

the corresponding network-dynamics CS reconstruction using (7) in Fig. 3. In the absence of network dynamics, we observe an improvement in reconstruction quality. Likewise, we find that the network-dynamics CS reconstruction using (7) yields a level of accuracy intermediate between the static and (9) reconstructions. Since (9) is a further linear approximation of (7) in the high-spike-frequency dynamical regime, it is reasonable to expect that some reconstruction accuracy will be lost. However, in Section 3.2, we observe that for more complex two-dimensional signals, the difference between the three reconstructions is much less pronounced, making the (9) networkdynamics CS reconstruction particularly useful for more realistic signal reconstructions. While some signal information appears not readily recovered through our particular compressive sensing of I\&F network dynamics, with more accurate input-output mappings, it may be possible to improve the quality of signal reconstructions. For this reason, an interesting area for future study would be to investigate the existence of more exact network input-output mappings applicable to network CS signal recovery. 
Comparing the frequency-domain representations of these reconstructions, we can more clearly understand their underlying differences and various levels of accuracy. In Fig. 4 (a)-(c), we plot the absolute value of the one-dimensional discrete Fourier transform of the original input signal and reconstructions using static CS, and network-dynamics CS corresponding to (7) and (9), respectively. For the most dominant frequencies, the three reconstructions are nearly identical to the original input. However, to reconcile for any minor discrepancies between the actual input signal and the output processed through network dynamics, the network-dynamics CS reconstructions introduce several additional low-amplitude, high-frequency components in order to satisfy constraint (11). We see that linearization (9) introduces several more such high-frequency components than nonlinear mapping (7), as can be observed for frequencies greater than 1, suggesting that more accurate mappings indeed have the potential to capture higher-order input signal information. Overall, we note that the general distribution of the high-amplitude, low-frequency components for the reconstructions is quite similar, yielding comparable corresponding input-signal reconstructions in the original nonsparse domain evidenced by Fig. 3. In fact, the proportion of dominant frequency components, with amplitude greater than 0.1, is nearly the same for all three reconstructions. Thus, both the static and network-dynamics CS recoveries can reveal similarly sparse and similarly accurate approximations of the original input signal.

While the compressive sensing solution appears to choose the reconstruction that best traces the input signal, we contrast that solutions to (9), which do not take into account any constraints on signal sparsity, do not resemble the injected input. We consider a combination of a particular solution to (9) and randomly chosen elements of the null space of connection matrix $B$, which defines the left-hand side of (9). Plotting two such naive solutions in Fig. 3, we observe that they clearly lack the coherent structure of the CS solution and are on average not even of the same order of magnitude as the actual input signal. To quantitatively assess the accuracy of the reconstruction, we measure the relative reconstruction error defined by

$$
\text { Error }=\left\|p-p_{\text {recon }}\right\| /\|p\|,
$$

where the Euclidean norm is defined $\|p\|=\sqrt{\sum_{i=1}^{n} p_{i}^{2}}$ and $p_{\text {recon }}$ is the reconstructed input signal. In the case of the CS solution, the relative reconstruction error is approximately $10 \%$, whereas the naive alternative re- 

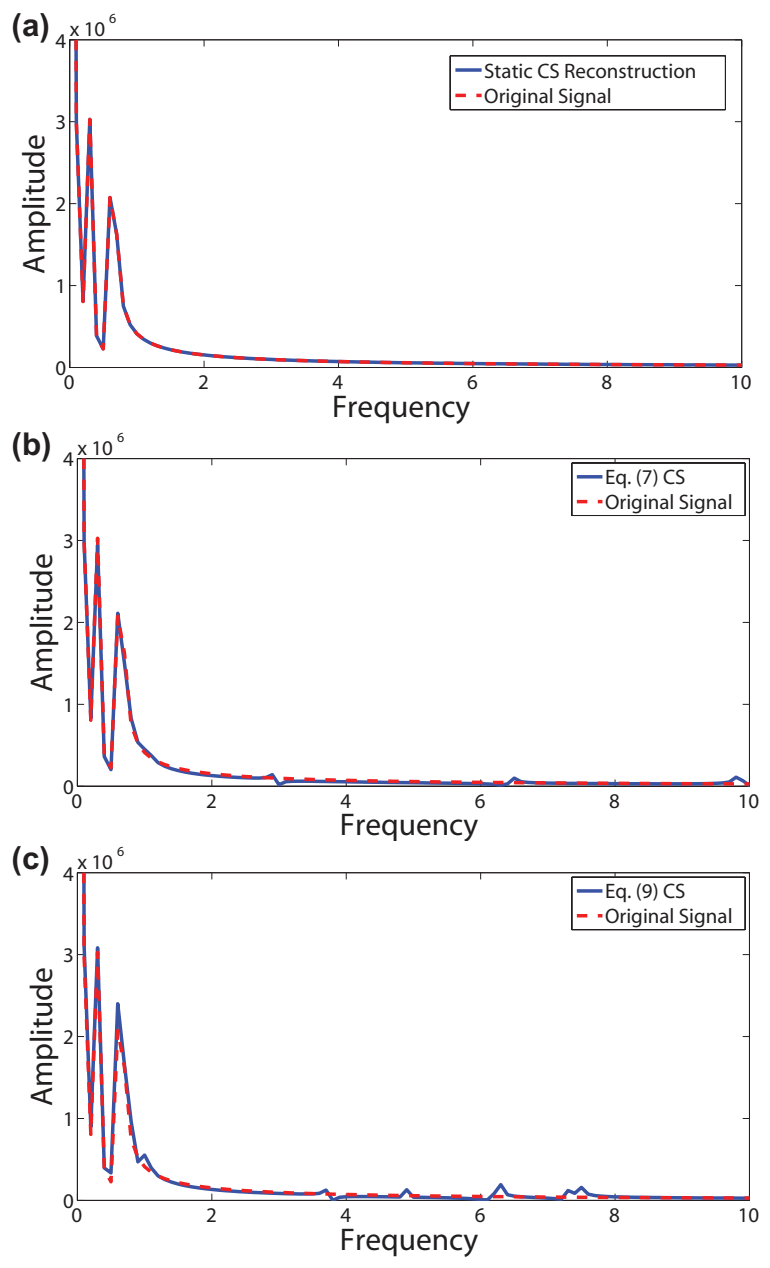

Figure 4: Frequency domain comparison. (a) Frequency domain representation of the static CS reconstruction (blue). (b) Frequency domain representation of the networkdynamics CS reconstruction using the nonlinear mapping given by (7) (blue). (c) Frequency domain representation of the network-dynamics CS reconstruction using the linearization given by (9) (blue). In each case, the absolute value of the discrete Fourier transform is displayed, using the one-dimensional input signal defined by (12). We plot each representation for frequencies less than 10 to zoom into the structure of the dominant frequencies. In each panel, we plot for comparison the frequency domain representation of the original signal (dashed red). The proportion of dominant frequency components, with amplitude greater than 0.1, for the static, (7), and (9) CS reconstructions are 0.0130, 0.0113 , and 0.0147 , respectively. Each simulated network is composed of $n=10000$ input neurons and $m=1000$ processing neurons. We choose the connection probability of $A$ to be 0.05 and $B$ to be 0.001 . 
constructions yield relative errors of more than 100\%. Hence, information appears well-preserved through the network dynamics, allowing a recognizable reconstruction even in the case where there is a factor of 10 less output measurements than input components.

\subsection{Two-Dimensional Input Signal Recovery}

We now consider CS reconstructions for four images differing in complexity, spatial patterning, and size. All four input images have a grey-scale pixel matrix representation with components taking on integer values indicating the light intensity of the signal at a given spatial location. The first two images are of size $100 \times 100$ pixels, and the last two are of size $200 \times 200$ pixels.

Figure 5 compares the original input image and the corresponding networkdynamics CS reconstruction using (9) for each case over a time-scale of $t_{f}=200 \mathrm{~ms}$. The first three images are listed in order of increasing complexity: a single disk, sequence of triangles, and a cameraman. The final image is an MRI brain scan of a patient with normal pressure hydrocephalus. In each case, we find that the network-dynamics CS reconstruction captures all spatial features very well with only minor perturbations in the grey-scale values of some individual pixels. The network-dynamics CS reconstruction appears to have more pronounced errors near edges or sharp transitions in pixel values, for which specific low-amplitude, high-frequency components of the image may have been lost through dynamics. For comparison, we also plot the corresponding static CS reconstruction using an identical sampling rate. While the static reconstructions do yield a smaller error, we find that qualitatively the dynamic and static CS reconstructions yield quite comparable results. The error for each reconstruction is listed in the caption of Fig. 5, demonstrating a comparable order of reconstruction accuracy for every image.

It is important to remark that, as in the case of compressive sensing applied to static images, better reconstructions are achievable in the context of larger images and those with more sparsity. The increase in pixel numbers allows for the reconstruction of finer-scale variations in grey-scale values, as in the case of the cameraman image. Likewise, using more output samples greatly increases the reconstruction accuracy. In Fig. 5, we also plot the corresponding network-dynamics CS reconstruction using an alternative reduction factor of 5, achieving markedly improved reconstructions. By in-

creasing the number of measured nodes, we indeed observe levels of accuracy 
(a)

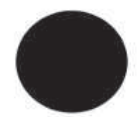

(e)

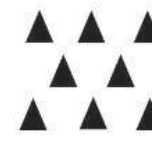

(i)

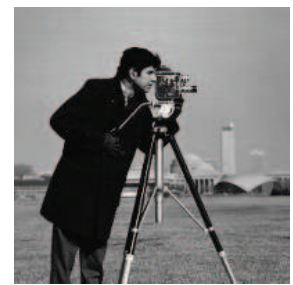

(m)

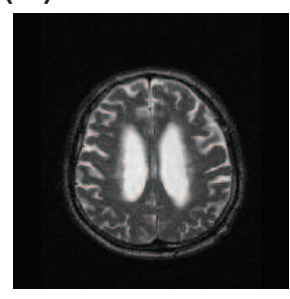

(b)

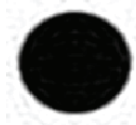

(f)

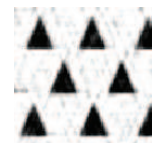

(j)

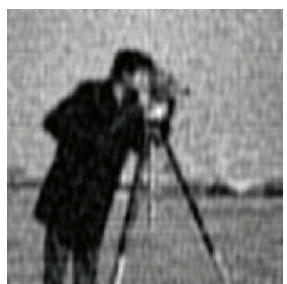

(n)

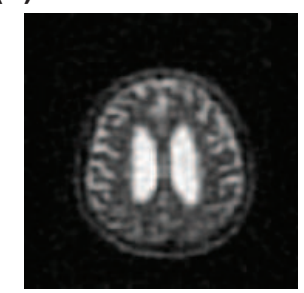

(c)

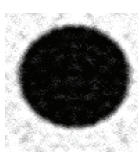

(g)

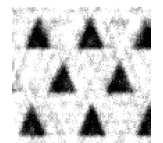

(k)

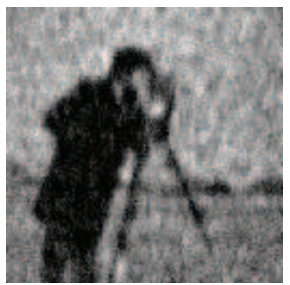

(o)

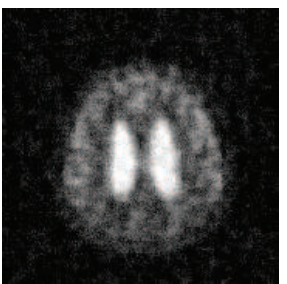

(d)

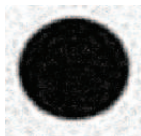

(h)

(I)
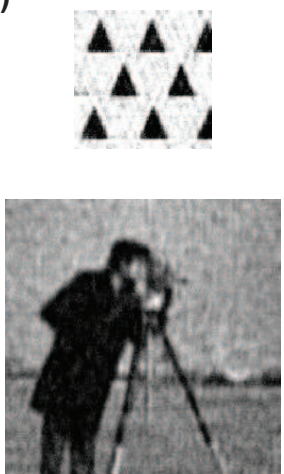

(p)

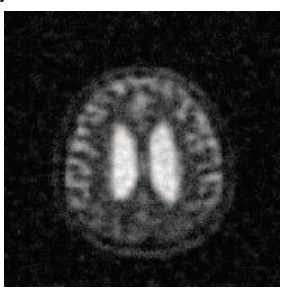

Figure 5: Reconstruction of two-dimensional input images. (a), (e), (i), (m): original input images; (b), (f), (j), (n): reconstructions using static CS with errors $0.0945,0.1111,0.1497,0.2081$ respectively; $(\mathrm{c}),(\mathrm{g}),(\mathrm{k}),(\mathrm{o})$ : reconstructions using network-dynamics CS and a $10: 1$ input neuron to processing neuron ratio with errors $0.1385,0.1692,0.2617$, respectively; $(\mathrm{d}),(\mathrm{h}),(\mathrm{l}),(\mathrm{p})$ : reconstructions using networkdynamics CS and a smaller 5 : 1 input neuron to processing neuron ratio with errors $0.1254,0.1345,0.1739,0.2422$ respectively. We choose the connection probability of $A$ to be 0.05 and $B$ to be 0.001 . Images (a) and (e) are of size $100 \times 100$ pixels and images (i) and $(\mathrm{m})$ are of size $200 \times 200$ pixels.

comparable to the static CS reconstruction. While in this particular work we use the two-dimensional discrete cosine transform to obtain relatively sparse image representations, we note that for some signals alternative transformations may yield even higher quality CS reconstructions. Nevertheless, the theory presented in Section 2 is generalizable to other sparse representations and may be adapted to recover other classes of signals.

Overall, we see that the quality of the static and network-dynamics CS reconstructions are more comparable in the realistic two-dimensional image 
case than for the simpler one-dimensional signal discussed in Section 3.1. This suggests that for more complex signals, with more dominant and varied frequency-components, the network-dynamics and static CS reconstructions may converge towards the same signal reconstruction for some input signals. Most likely, the frequency components for more complex signals missed by the network-dynamics CS reconstruction but captured by the static CS recovery do not contribute as heavily to the overall features in the signal, and therefore they do not significantly alter the reconstruction quality. Similarly, we comment that the reconstruction quality corresponding to the (7) network-dynamics CS reconstruction is nearly identical to that of linearization (9) since the significant modes are nearly equally captured by the two input-output mappings. We therefore conclude that even for highly-detailed input signals measured using a very small number of output samples, the network-dynamics CS reconstruction is quite robust and naturally generalizes conventional static compressive sensing.

\section{Discussion}

Since the discovery of compressive sensing theory, the field of signal processing has been revolutionized and rapidly expanded. In this work, we have presented a new extension of CS to the recovery of signals driving I\&F neuronal network dynamics. While CS is typically applied to static linear systems, biological network dynamics are generally nonlinear and inherently non-static. Despite this, for a class of I\&F networks with pulse-coupled nonlinear dynamics, we derived a linear map between an unknown network input with a large number of components and the measured output processed through a relatively small number of nodes. Then, we presented a detailed framework for reconstructing the network input, applying CS theory to the measured network dynamics and derived linear input-output relation. Finally, we used our methodology to recover diverse input signals, yielding rather accurate reconstructions in each case. Comparing static CS recovery of sampled signals to the reconstruction of the same signals processed through I\&F network dynamics, we demonstrated that input information is well-preserved through realistic network dynamics even when such networks have significantly fewer nodes than input signal components.

This connection between CS and network dynamics suggests a new direction for both biological and signal processing research. While we present a formal derivation of the network linear input-output map for one particular 
class of I\&F networks, we have yet to make a similar argument for more general network dynamics. Likewise, akin to conventional CS analysis [1, 3], it would be interesting to analytically determine the probability of successful signal recovery via network-dynamics CS. In addition, it would be informative to investigate the characteristics of network dynamics that best encode input signal information. We expect that by using input sampling techniques common in visual systems, such as receptive field structure, improved reconstruction of network inputs may be possible in our framework [68, 69].

It is important to note that network-dynamics CS can be applied to experimentally observed physical networks in addition to numerically simulated networks. For example, the underlying linear structure of a real-world network could be recovered via first-order Wiener kernel analysis, upon which CS theory could then be applied to the measured network output [70, 71]. With respect to image segmentation in particular, we expect that since image information is well-preserved through I\&F network dynamics utilizing far fewer nodes than image pixels, it may not be necessary to use a $1: 1$ ratio of nodes to pixels as typically done in image processing. Instead, it may be possible to use CS theory to dramatically reduce the number of nodes necessary for successful segmentation or improve the quality of more general image-processing.

\section{Acknowledgements}

This work was supported by NYU Abu Dhabi Institute G1301 (V.B., G.K., D.Z., D.C.), NSF DMS-0636358 (V.B.), NSFC-91230202, Shanghai Rising-Star Program-15QA1402600 (D.Z.), NSF DMS-1009575 (D.C.), and Shanghai 14JC1403800 (D.C., D.Z.).

[1] E. J. Candes, J. K. Romberg, T. Tao, Stable signal recovery from incomplete and inaccurate measurements, Communications on Pure and Applied Mathematics 59 (8) (2006) 1207-1223.

[2] E. J. Candes, M. B. Wakin, An Introduction To Compressive Sampling, Signal Processing Magazine, IEEE 25 (2) (2008) 21-30.

[3] D. L. Donoho, Compressed sensing, IEEE Trans. Inform. Theory 52 (2006) 1289-1306. 
[4] W. Yin, S. Osher, D. Goldfarb, J. Darbon, Bregman iterative algorithms for 1-minimization with applications to compressed sensing, SIAM J. Imaging Sci (2008) 143-168.

[5] M. Figueiredo, R. Nowak, S. Wright, Gradient projection for sparse reconstruction: Application to compressed sensing and other inverse problems, IEEE Journal of Selected Topics in Signal Processing 1 (4) (2008) 586-597.

[6] R. Baraniuk, Compressive sensing, IEEE Signal Processing Mag (2007) $118-120$.

[7] D. Gross, Y. K. Liu, S. T. Flammia, S. Becker, J. Eisert, Quantum state tomography via compressed sensing, Phys. Rev. Lett. 105 (15) (2010) 150401.

[8] M. Lustig, D. Donoho, J. M. Pauly, Sparse MRI: The application of compressed sensing for rapid MR imaging, Magn. Reson. Med. 58 (6) (2007) 1182-1195.

[9] W. Dai, M. A. Sheikh, O. Milenkovic, R. G. Baraniuk, Compressive sensing DNA microarrays, J. Bioinform. Syst. Biol. (2009) 162824.

[10] C. R. Berger, Z. Wang, J. Huang, S. Zhou, Application of compressive sensing to sparse channel estimation, Comm. Mag. 48 (11).

[11] M. A. Herman, T. Strohmer, High-resolution radar via compressed sensing, Trans. Sig. Proc. 57 (6) (2009) 2275-2284.

[12] J. Bobin, J.-L. Starck, R. Ottensamer, Compressed sensing in astronomy., J. Sel. Topics Signal Processing 2 (5) (2008) 718-726.

[13] A. N. Burkitt, A review of the integrate-and-fire neuron model: I. homogeneous synaptic input., Biol Cybern 95 (1) (2006) 1-19. doi:10.1007/s00422-006-0068-6.

[14] R. E. Mirollo, S. H. Strogatz, Synchronization of pulse-coupled biological oscillators, SIAM Journal on Applied Mathematics 50 (6) (1990) 16451662 . 
[15] D. Somers, S. Nelson, M. Sur, An emergent model of orientation selectivity in cat visual cortical simple cells, Journal of Neuroscience 15 (1995) 5448-5465.

[16] W. Mather, M. R. Bennett, J. Hasty, L. S. Tsimring, Delay-induced degrade-and-fire oscillations in small genetic circuits, Phys. Rev. Lett. 102 (6) (2009) 068105.

[17] A. Corral, C. J. Pérez, A. Díaz-Guilera, A. Arenas, Self-organized criticality and synchronization in a lattice model of integrate-and-fire oscillators., Phys Rev Lett 74 (1) (1995) 118-121.

[18] R. Mirollo, S. Strogatz, Synchronization of Pulse-Coupled Biological Oscillators, SIAM Journal on Applied Mathematics 50 (6) (1990) 16451662 .

[19] J. Buck, Synchronous Rhythmic Flashing of Fireflies. II., The Quarterly Review of Biology 63 (3) (1988) 265-289.

[20] D. Cai, A. Rangan, D. McLaughlin, Architectural and synaptic mechanisms underlying coherent spontaneous activity in V1, Proc. Nat'l Acad. Sci (USA) 102 (2005) 5868-5873.

[21] A. V. Rangan, D. Cai, D. W. McLaughlin, Modeling the spatiotemporal cortical activity associated with the line-motion illusion in primary visual cortex, Proc. Natl. Acad. Sci. USA 102 (52) (2005) 18793-18800.

[22] V. J. Barranca, D. C. Johnson, J. L. Moyher, J. P. Sauppe, M. S. Shkarayev, G. Kovačič, D. Cai, Dynamics of the exponential integrateand-fire model with slow currents and adaptation, J Comput Neurosci.

[23] L. Zhao, E. N. Macau, A network of dynamically coupled chaotic maps for scene segmentation, IEEE Trans Neural Netw 12 (6) (2001) 13751385 .

[24] S. R. Campbell, D. L. Wang, C. Jayaprakash, Synchrony and desynchrony in integrate-and-fire oscillators, Neural Comput 11 (7) (1999) 1595-1619.

[25] T. Lindblad, J. M. Kinser, Image processing using pulse-coupled neural networks., Perspectives in neural computing, Springer, 1998. 
[26] Z. Wang, Y. Ma, F. Cheng, L. Yang, Review of pulse-coupled neural networks, Image and Vision Computing 28 (1) (2010) 5-13. doi:10.1016/j.imavis.2009.06.007.

[27] Y. Ma, D. Lin, B. Zhang, C. Xia, A novel algorithm of image enhancement based on pulse coupled neural network time matrix and rough set., in: J. Lei (Ed.), FSKD (3), IEEE Computer Society, 2007, pp. 86-90.

[28] H. S. Ranganath, G. Kuntimad, Object detection using pulse coupled neural networks, IEEE Trans Neural Netw 10 (3) (1999) 615-620.

[29] K. Waldemark, T. Lindblad, V. Beanovi, J. L. L. Guillen, P. L. Klingner, Patterns from the sky: Satellite image analysis using pulse coupled neural networks for pre-processing, segmentation and edge detection., Pattern Recognition Letters 21 (3) (2000) 227-237.

[30] R. C. Muresan, Pattern recognition using pulse-coupled neural networks and discrete fourier transforms., Neurocomputing 51 (2003) 487-493.

[31] X. Gu, Feature extraction using unit-linking pulse coupled neural network and its applications., Neural Processing Letters 27 (1) (2008) 2541.

[32] R. P. Broussard, S. K. Rogers, M. E. Oxley, G. L. Tarr, Physiologically motivated image fusion for object detection using a pulse coupled neural network, IEEE Trans Neural Netw 10 (3) (1999) 554-563.

[33] D. J. Field, What is the goal of sensory coding?, Neural Computation 6 (4) (1994) 559-601. doi:10.1162/neco.1994.6.4.559.

[34] C. E. Shannon, Communication in the Presence of Noise, Proceedings of the IRE 37 (1) (1949) 10-21.

[35] D. J. Tolhurst, Y. Tadmor, T. Chao, Amplitude spectra of natural images, Ophthalmic. Physiol. Opt. 12 (2) (1992) 229-232.

[36] B. A. Olshausen, D. J. Field, Natural image statistics and efficient coding, Network 7 (2) (1996) 333-339.

[37] E. P. Simoncelli, B. A. Olshausen, Natural image statistics and neural representation, Annu. Rev. Neurosci. 24 (2001) 1193-1216. 
[38] A. M. Bruckstein, D. L. Donoho, M. Elad, From sparse solutions of systems of equations to sparse modeling of signals and images, SIAM Review 51 (1) (2009) 34-81.

[39] J. A. Tropp, A. C. Gilbert, Signal Recovery From Random Measurements Via Orthogonal Matching Pursuit, IEEE Transactions on Information Theory 53 (12) (2007) 4655-4666.

[40] D. L. Donoho, Y. Tsaig, Fast solution of $1_{1}$-norm minimization problems when the solution may be sparse, IEEE Transactions on Information Theory 54 (11) (2008) 4789-4812.

[41] M. Elad, A. M. Bruckstein, A generalized uncertainty principle and sparse representation in pairs of bases., IEEE Transactions on Information Theory 48 (9) (2002) 2558-2567.

[42] D. Zhou, A. V. Rangan, D. W. McLaughlin, D. Cai, Spatiotemporal dynamics of neuronal population response in the primary visual cortex, Proc. Natl. Acad. Sci. U.S.A. 110 (23) (2013) 9517-9522.

[43] S. Strogatz, Exploring complex networks, Nature 410 (2001) 268-276.

[44] S. N. Dorogovtsev, J. F. F. Mendes, Evolution of networks, Advances in Phys. 51 (2002) 1079-1187.

[45] M. Newman, The structure and function of complex networks, SIAM Rev. 45 (2003) 167-256.

[46] S. Boccaletti, V. Latora, Y. Moreno, M. Chavez, D.-U. Hwang, Complex networks: Structure and dynamics, Physics Reports 424 (2006) 175-308.

[47] O. Omidvar, J. Dayhoff (Eds.), Neural Networks and Pattern Recognition, Academic Press, San Diego, Chestnut Hill, London, 1997.

[48] J. Johnson, M. Padgett, O. Omidvar, Guest editorial overview of pulse coupled neural network (pcnn) special issue, Neural Networks, IEEE Transactions on 10 (3) (1999) 461-463.

[49] W. Gerstner, W. Kistler, Spiking Neuron Models - Single Neurons, Populations, Plasticity, Cambridge University Press, New York, 2002. 
[50] A. N. Burkitt, A review of the integrate-and-fire neuron model: Ii. inhomogeneous synaptic input and network properties., Biol Cybern 95 (2) (2006) 97-112. doi:10.1007/s00422-006-0082-8.

[51] A. Barrat, M. Barthélemy, A. Vespignani, Dynamical Processes on Complex Networks, Cambridge University Press, Cambridge, New York, 2008.

[52] T. Lindblad, J. M. Kinser, Image Processing Using Pulse-Coupled Neural Networks, Springer, Berlin, Heidelberg, 2010.

[53] Y. Ma, K. Zhan, Z. Wang, Applications of Pulse-Coupled Neural Networks, Springer, New York, 2010.

[54] H. Markram, J. Lubke, M. Frotscher, A. Roth, B. Sakmann, Physiology and anatomy of synaptic connections between thick tufted pyramidal neurones in the developing rat neocortex., J Physiol 500 ( Pt 2) (1997) 409-440.

[55] A. Mason, A. Nicoll, K. Stratford, Synaptic transmission between individual pyramidal neurons of the rat visual cortex in vitro, J. Neurosci. 11 (1) (1991) 72-84.

[56] E. Ganmor, R. Segev, E. Schneidman, The architecture of functional interaction networks in the retina, J. Neurosci. 31 (8) (2011) 3044-3054.

[57] R. Brette, M. Rudolph, T. Carnevale, M. Hines, D. Beeman, J. M. Bower, M. Diesmann, A. Morrison, P. H. Goodman, F. C. Harris Jr, M. Zirpe, T. Natschlager, D. Pecevski, B. Ermentrout, M. Djurfeldt, A. Lansner, O. Rochel, T. Vieville, E. Muller, A. P. Davison, S. E. Boustani, A. Destexhe, Simulation of networks of spiking neurons: A review of tools and strategies, J. Comput. Neurosci. 23 (3) (2007) 349398.

[58] K. A. Newhall, G. Kovačič, P. R. Kramer, D. Cai, Cascade-induced synchrony in stochastically-driven neuronal networks, Phys. Rev. E 82 (2010) 041903.

[59] D. A. McCormick, B. W. Connors, J. W. Lighthall, D. A. Prince, Comparative electrophysiology of pyramidal and sparsely spiny stellate neurons of the neocortex, J. Neurophysiol. 54 (4) (1985) 782-806. 
[60] M. Shelley, D. McLaughlin, R. Shapley, J. Wielaard, States of high conductance in a large-scale model of the visual cortex, J. Comp. Neurosci. 13 (2002) 93-109.

[61] G. Kovačič, L. Tao, A. V. Rangan, D. Cai, Fokker-planck description of conductance-based integrate-and-fire neuronal networks, Phys. Rev. E 80 (2009) 021904.

[62] K. Amano, N. Goda, S. Nishida, Y. Ejima, T. Takeda, Y. Ohtani, Estimation of the timing of human visual perception from magnetoencephalography, J. Neurosci. 26 (15) (2006) 3981-3991.

[63] S. Ando, Y. Yamada, M. Kokubu, Reaction time to peripheral visual stimuli during exercise under hypoxia, J. Appl. Physiol. 108 (5) (2010) $1210-1216$.

[64] A. V. Rangan, D. Cai, Maximum-entropy closures for kinetic theories of neuronal network dynamics, Phys. Rev. Lett. 96 (17) (2006) 178101.

[65] D. Cai, L. Tao, M. Shelley, D. McLaughlin, An effective representation of fluctuation-driven neuronal networks with application to simple \& complex cells in visual cortex, Pro. Nat. Acad. Sci. (USA) 101 (2004) 7757-7762.

[66] E. Cinlar, Superposition of point processes, in: P. Lewis (Ed.), Stochastic Point Processes: Statistical Analysis, Theory, and Applications, Wiley, New York, NY, 1972, pp. 549-606.

[67] M. T. Heath, Scientific Computing: An Introductory Survey, McGrawHill, New York, 2002.

[68] T. N. Wiesel, Receptive fields of ganglion cells in the cat's retina, J Physiol 153 (1960) 583-594.

[69] D. H. Hubel, T. N. Wiesel, Receptive fields of optic nerve fibres in the spider monkey., J Physiol 154 (1960) 572-580.

[70] N. Wiener, Nonlinear Problems in Random Theory, Technology Press Research Monographs, The Technology Press of Massachusetts Institute of Technology and John Wiley \& Sons, Cambridge, 1958. 
[71] J. Victor, Nonlinear systems analysis in vision: Overview of kernel methods, in: B. N. R.B. Pinter (Ed.), Nonlinear vision: Determination of Neural Receptive Fields, Function, and Networks, CRC Press, Boca Raton, 1992, pp. 1-37. 